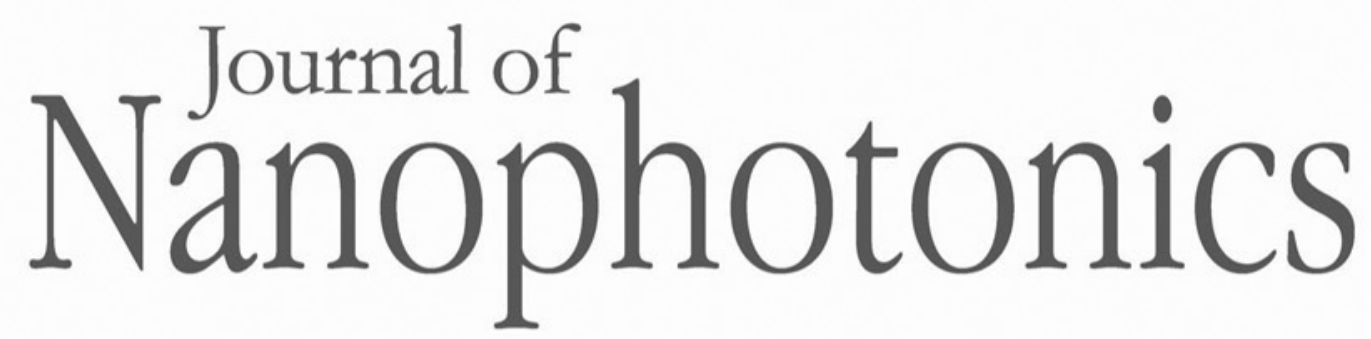

Nanophotonics.SPIEDigitalLibrary.org

\title{
2019 List of Reviewers
}


The Journal of Nanophotonics would like to sincerely thank the following individuals who served as reviewers in 2019. The success of our publication hinges on the voluntary contributions of time and energy put forth by these professionals.

Venu Gopal Achanta

Ali Adibi

Kofi Adu

Imad H. Agha

Edward Aifer

Naznin Akter

Musab A. M. Al-Tarawni

Kirill Alekseev

Arafa Aly

Soosaimanickam

Ananthakumar

Muthiah Annamalai

Margarida Barroso

Konstantin Batrakov

Elyas Bayati

Can Bayram

Feroza Begum

Anne Berends

Indrasen Bhattacharya

Nikita Bityrin

Mohammad Bolorizadeh

Jiten Boruah

Mongi Bouaicha

Matthew Brennesholtz

Paulo Caldas

Ayan Chakrabarty

Che Chen

Kai Chen

Lin Chen

Shiyi Chen

Alan Chin

Ahmad Darweesh

Anurup Datta

Ignacio Del Villar

Mai Desouky

Sai Cong Doanh

Denis Donlagic

Hendry Elim

Ramin Emadi

Jonathan Fan

Tianren Fan

Muhammad Faryad

Kaijun Feng

Stavroula Foteinopoulou

Chien-Chung Fu

Jing Gao

Yang Gao

Sergey Gaponenko

Shayan Garani

Amir Ghobadi

Arnold Goldberg

Hong Guo

Dongheon $\mathrm{Ha}$

Mohsin Habib

Terefe Habteyes

Haroldo Hattori
Arnab Hazari

Ravi Hegde

Roland Himmelhuber

Fei Huang

John Hubbs

Niranjan llawe

Daehwan Jung

Saulius Juodkazis

Kiyoshi Kanie

Akash Kannegulla

Tommi Kaplas

Anshika Kapur

Jyoti Kedia

Yashar Kiarashinejad

Kenji Kintaka

Naoki Kishimoto

Victor Kleshch

Viktor Koroteev

Victor Krivenkov

Mitsutaka Kumakura

Yin-Chieh Lai

Akhlesh Lakhtakia

Khai Le

Joonhee Lee

Guangyuan Li

Rujiang Li

Xiaofeng $\mathrm{Li}$

Tong Lin

Joerg Lindner

Chaofeng Liu

Kristof Lodewijks

Prasanta Mandal

Geza Mark

Arthur McGurn

Claiborne McPheeters

Masood Mehrabian

Hosam Mekawey

Sabina Merlo

Akio Mizutani

Dmitri Mogilevtsev

Ramesh Mohan

Antoine Moreau

Dariusz Moszynski

Venkatesha Muniswamy

Terence Musho

Aparna Nair

Abdoulaye Ndao

Michael P. Nielsen

Shinpei Ogawa

Hamza Otmani

Laurent Oyhenart

Liang Pan

Hamed Pezeshki

Pierpaolo Porta

Andrei Postnikov

Klaus Rademann
Jelena Radovanovic

Kalyani Rajendran

lleana Rau

Srikanth Ravipati

Balusamy Renganathan

Jorge-Alejandro Reyes-

Esqueda

Peter Rolfe

Filippo Romanato

Sandipta Roy

Halina Rubinsztein-Dunlop

Patrick Rufangura

Shlomo Ruschin

Alp Oral Salman

Sarath Samudrala

Andrew Sarangan

S. Saravanan

Hayk Sarkisyan

Gabby Sarusi

Malathi Sathish

Salvatore Scaglione

Tigran Sedrakyan

Taner Sengor

Guangcun Shan

Anuj Sharma

Divya Sharma

Maxim Shcherbakov

Weichuan Shih

Saeid Shojaei

Mikhail Shuba

Thomas Sondergaard

Alberto Sposito

Guru Subramanyam

Huiyuan Sun

Charles Surya

Yuri Svirko

Mohammad Taghinejad

Litty Thekkekara

Gene Tsvid

Takashi Uchino

Vijayakumar Venugopal

Georgios Veronis

Slobodan Vukovic

Rami Wahsheh

Benxin Wang

Letian Wang

Mingqing Wang

Tianyi Wang

Daniel Wasserman

Guillaume Weick

Debra Weidanz

Shuyuan Xiao

Peipeng $X u$

Yunlu $\mathrm{Xu}$

Keisaku Yamane

Hai Yan 
2019 List of Reviewers

Daquan Yang

Fan Ye

Fei Yi

Nobuhiko Yokoshi

Qianhuan Yu
Ferdows B. Zarrabi

Xin Zhang

Qiancheng Zhao

Yi Zhao

Jiajiu Zheng
Changhe Zhou

Ru Zhou

Salah Zohary

Shengli Zou 